\title{
Decomposition of a recursive family of polynomials
}

\author{
Andrej Dujella and Ivica Gusić
}

\begin{abstract}
We describe decomposition of polynomials $f_{n}:=f_{n, B, a}$ defined by$$
f_{0}:=B, f_{1}(x):=x, f_{n+1}(x)=x f_{n}(x)-a f_{n-1}(x)
$$

where $B$ and $a$ are rational numbers. We also present an application to related Diophantine equations.

\section{Introduction}

It is an essential question on the polynomial Diophantine equation $f(x)=$ $g(y)$ to ask whether this equation has finitely or infinitely many integer solutions. In 2000, Bilu and Tichy [3] obtained a completely explicit finiteness criterion. Their result generalizes a previous one due to Schinzel [14, Theorem 8], who gave a finiteness criterion under the assumption $\operatorname{gcd}(\operatorname{deg} f, \operatorname{deg} g)$ $=1$. These criteria are closely connected with decomposability properties of the polynomials $f$ and $g$. A polynomial $f \in \mathbb{C}[x]$ is called indecomposable (over $\mathbb{C}$ ) if $f=g \circ h, g, h \in \mathbb{C}[x]$ implies $\operatorname{deg} g=1$ or $\operatorname{deg} h=1$. Two decompositions of $f$, say $f=g_{1} \circ h_{1}$ and $f=g_{2} \circ h_{2}$ are equivalent if there exists a linear function $L$ such that $g_{2}=g_{1} \circ L, h_{2}=L^{-1} \circ h_{1}$ (see [14, pp. $14-15])$.

To formulate the criterion of Bilu and Tichy, we have to define five types of standard pairs $(f(x), g(x))$. In what follows, $a$ and $b \in \mathbb{Q} \backslash\{0\}, m$ and $n$ are positive integers, and $p(x)$ is a non-zero polynomial.

A standard pair of the first kind is a pair of the form $\left(x^{m}, a x^{r} p(x)^{m}\right)$, or switched, $\left(a x^{r} p(x)^{m}, x^{m}\right)$ where $0 \leq r<m, \operatorname{gcd}(r, m)=1$ and $r+\operatorname{deg} p(x)>$ 0 . A standard pair of the second kind is $\left(x^{2},\left(a x^{2}+b\right) p(x)^{2}\right)$ (or switched).

2000 Mathematics Subject Classification 11B39, 11C08, $12 \mathrm{E} 05$.

The authors were supported by the Ministry of Science, Education and Sports, Republic of Croatia, grant 0037110. 
Denote by $D_{m, a}(x)$ the $m$-th Dickson polynomial, defined by

$$
D_{m, a}(z+a / z)=z^{m}+(a / z)^{m} .
$$

A standard pair of the third kind is $\left(D_{m, a^{n}}(x), D_{n, a^{m}}(x)\right)$, where $\operatorname{gcd}(m, n)$ $=1$. A standard pair of the fourth kind is $\left(a^{-m / 2} D_{m, a}(x),-b^{-n / 2} D_{n, b}(x)\right)$, where $\operatorname{gcd}(m, n)=2$.

A standard pair of the fifth kind is $\left(\left(a x^{2}-1\right)^{3}, 3 x^{4}-4 x^{3}\right)$ (or switched).

Please note that the $a, b$ appearing in the definitions of standard pairs are different from those used in the recurrences of $f_{n, B, a}$, resp. $f_{m, C, b}$.

ThEOREM $1([3])$ Let $f(x), g(x) \in \mathbb{Q}[x]$ be non-constant polynomials. Then the following two assertions are equivalent.

(a) The equation $f(x)=g(y)$ has infinitely many rational solutions with a bounded denominator.

(b) We have $f=\varphi \circ f_{1} \circ \lambda$ and $g=\varphi \circ g_{1} \circ \mu$, where $\lambda(x), \mu(x) \in \mathbb{Q}[x]$ are linear polynomials, $\varphi(x) \in \mathbb{Q}[x]$, and $\left(f_{1}(x), g_{1}(x)\right)$ is a standard pair over $\mathbb{Q}$ such that the equation $f_{1}(x)=g_{1}(y)$ has infinitely many rational solutions with a bounded denominator.

The criterion of Bilu and Tichy has been already applied to several Diophantine equations of the form $f_{n}(x)=g_{m}(y)$, where $\left(f_{n}\right)$ and $\left(g_{n}\right)$ are sequences of classical polynomials (see $[1,2,7,10,11,12,13,18,19,20])$. In particular, in [7], the equation $G_{m}(x)=G_{n}(y)$ was considered, where $\left(G_{n}\right)$ is the sequence of generalized Fibonacci polynomials defined by $G_{0}(x)=0$, $G_{1}(x)=1, G_{n+1}=x G_{n}(x)-a G_{n-1}$ for $n \geq 1$. In [5] (see also [6]), we obtained the following general criteria for indecomposability of polynomials, in terms of the degree and two leading coefficients.

\section{THEOREM 2}

i) [5, Theorem 2.2] Let $f(x)=x^{n}+u x^{n-1}+\cdots \in \mathbb{Z}[x]$. If $\operatorname{gcd}(u, n)=1$, then $f$ is indecomposable.

ii) [5, Corollary 3.1] Let $f(x)=d x^{n}+u x^{n-2}+\cdots \in \mathbb{Z}[x]$ be an odd polynomial. If $\operatorname{gcd}(d u, n)=1$, then $f$ is indecomposable.

iii) [5, Theorem 3.1] Let $f(x)=d x^{2 n}+u x^{2 n-2}+\cdots \in \mathbb{Z}[x]$ be an even polynomial and define $g(x)=f(\sqrt{x})$. Assume that $\operatorname{gcd}(u, n)=1$. Then every decomposition of $f$ is equivalent to one of the following decompositions: 
(a) $f(x)=g\left(x^{2}\right)$,

(b) $f(x)=\left(x P\left(x^{2}\right)\right)^{2}$.

The case (b) appears if and only if $g(x)=x P(x)^{2}$ for some polynomial $P(x) \in \mathbb{Z}[x]$.

In this paper, we will generalize the results from [7], by applying Theorems 1 and 2 to a more general family of polynomials $f_{n}:=f_{n, B, a}$ defined by

$$
f_{0}:=B, f_{1}(x):=x, f_{n+1}(x)=x f_{n}(x)-a f_{n-1}(x)
$$

where $B$ and $a$ are rational numbers. We prove that, under certain mild technical conditions, the polynomials $f_{n}$ are (almost) indecomposable (Theorem 3) and have only simple roots (Theorem 4). These results allow us to apply the criterion of Bilu and Tichy in order to prove that, under the same technical conditions, the equation $f_{n, B, a}(x)=f_{m, C, b}(y)$ has only finitely many rational solutions with a bounded denominator (Theorem 5 ).

\section{The polynomials $f_{n, B, a}$}

Recall that Dickson polynomials $D_{n, a}$ satisfy the recurrence $D_{0, a}=2, D_{1, a}=$ $x, D_{n+1, a}(x)=x D_{n, a}(x)-a D_{n-1, a}(x)$.

The following properties of Dickson polynomials are well-known:

$$
\begin{gathered}
D_{n, a}(x)=\sum_{k \leq \frac{n}{2}} \frac{n}{n-k}\left(\begin{array}{c}
n-k \\
k
\end{array}\right)(-a)^{k} x^{n-2 k} \\
=x^{n}-n a x^{n-2}+\frac{n(n-3)}{2 !} a^{2} x^{n-4}-\frac{n(n-4)(n-5)}{3 !} a^{3} x^{n-6}+\cdots \\
D_{n, b^{2} a}(b x)=b^{n} D_{n, a}(x) .
\end{gathered}
$$

Now we will consider the polynomials $f_{n}:=f_{n, B, a}$ defined by (1.1). We first recall some special cases:

(a) $f_{n, B, 0}(x)=x^{n}$, for $n \geq 1$.

(b) $f_{n, 2, a}=D_{n, a}$ - the Dickson polynomials.

(c) $f_{n, 1, a}=G_{n+1}$ (from [7]), especially $f_{n, 1,1}=F_{n+1}$ - the Fibonacci polynomials. 
LEMMA 1 We have:

i) $f_{n, B, a}(x)=\frac{B}{2}\left(q^{n}+\left(\frac{a}{q}\right)^{n}\right)+\frac{2-B}{2} \frac{x}{\sqrt{x^{2}-4 a}}\left(q^{n}-\left(\frac{a}{q}\right)^{n}\right)$, where $q:=\frac{x+\sqrt{x^{2}-4 a}}{2}$.

ii) $f_{n, B, a}(x)=\sum_{k \leq \frac{n}{2}}\left(\frac{B}{2} \frac{n}{n-k}\left(\begin{array}{c}n-k \\ k\end{array}\right)-\frac{B-2}{2}\left(\begin{array}{c}n-k-1 \\ k\end{array}\right)\right)(-a)^{k} x^{n-2 k}$

iii) $f_{n, B, b^{2} a}(b x)=b^{n} f_{n, B, a}(x)$, for each complex number $b \neq 0$. Especially, if $a \neq 0$ and $\sqrt{a}$ is a fixed square root of $a$, then $f_{n, B, a}(x)=(\sqrt{a})^{n} f_{n, B, 1}\left(\frac{x}{\sqrt{a}}\right)$.

Proof. Solving the recurrence (1.1), we obtain i). It is easy to see, using $[9$, formulas (5.74) and (5.75)], that i) implies ii). It is clear that iii) follows from ii).

Note that the coefficient of $x^{n-2 k}$ may be written as

$$
\frac{n+(B-2) k}{n-k}\left(\begin{array}{c}
n-k \\
k
\end{array}\right)(-a)^{k}
$$

i.e. as

$$
\left(\left(\begin{array}{c}
n-k \\
k
\end{array}\right)+(B-1)\left(\begin{array}{c}
n-k-1 \\
k-1
\end{array}\right)\right)(-a)^{k}
$$

Note also that ii) is a generalization of the corresponding formula for $D_{n, a}$. Especially,

$$
f_{n, B, a}(x)=x^{n}-(B+n-2) a x^{n-2}+\frac{(n+2(B-2))(n-3)}{2 !} a^{2} x^{n-4}+\ldots
$$

Note that the polynomials $f_{n, B, a}$ are odd (for $n$ odd), resp. even (for $n$ even).

In the sequel, we set $B:=\frac{\mu}{d}$, where $\mu, d$ are co-prime integers and $d>0$. If $B=0$, then we set $\mu=0$ and $d=1$.

Theorem 3 Put $f_{n}=f_{n, B, a}$ and $H_{n}(x):=f_{2 n}(\sqrt{x})$. Assume that $a \neq 0$, $\operatorname{gcd}(d, n)=1$ and $\operatorname{gcd}(\mu-2 d, n)=1$. Then:

i) If $n$ is odd, then $f_{n}$ is indecomposable.

ii) $f_{2 n}(x)=H_{n}\left(x^{2}\right)$ is the unique (up to the equivalence) decomposition of $f_{2 n}$.

Proof. 
i) By Lemma 1 iii), $f_{n, B, a}$ is indecomposable if and only if $f_{n, B, 1}$ is indecomposable (provided $a \neq 0$ ). However $f_{n, B, 1}$ is indecomposable if and only if $d f_{n, B, 1}$ is indecomposable. By (2.3), $d f_{n, B, 1}$ is a polynomial over integers, and so, by Theorem 2 ii) and (2.4), it is indecomposable provided $n$ is odd, $\operatorname{gcd}(d, n)=1$ and $\operatorname{gcd}(\mu-2 d, n)=1$.

ii) Using a similar argument as in i), we see that the decompositions of $f_{2 n, B, a}$ correspond to the decompositions of $d f_{2 n, B, 1}$ (for $a \neq 0$ ). Also, if $a \neq 0$, then $H_{n, B, a}$ is decomposable if and only if $H_{n, B, 1}$ is decomposable. By Theorem 2 iii), the uniqueness of the decomposition may fail only if $d H_{n}(x)=x P(x)^{2}$ for a polynomial $P$. Then $n$ is odd and $H_{n}(0)=0$. Since $H_{n}(0)=\frac{2 n+(B-2) n}{2 n-n}\left(\begin{array}{c}2 n-n \\ n\end{array}\right)(-a)^{n}$ and $a \neq 0$, we see that $B=0$.

However, if $f_{2 n, 0, a}=x^{2} P\left(x^{2}\right)^{2}$, then the relation $f_{k, 0, a}=x f_{k-1,1, a}$ implies that the polynomial $f_{2 n-1,1, a}$ has a double root, which is impossible by Theorem 4 below.

REMARK 1 As a consequence of Theorem 3 we see that if $a \neq 0$, and $B=$ 1 or $B=3$, then $f_{n, B, a}$ is indecomposable (for $n$ odd), and $f_{n, B, a}(x)=$ $H_{\frac{n}{2}, B, a}\left(x^{2}\right)$ is the unique decomposition up to the equivalence (for $n$ even). For $B=1$ it was proved in [7] by other methods.

Also, we see that for $a \neq 0$ the following is valid: if $n$ is odd then $f_{n, \pm 2^{r}+2, a}$ is indecomposable for all $r \in \mathbf{N} \cup\{0\}$.

If $B=2$ then $\operatorname{gcd}(n+B-2, n)=n$, which is compatible with fact that Dickson polynomials are decomposable.

Example $1 A$ polynomial $H_{n, B, a}$ may be decomposable even if $B \neq 2$. For example,

$$
f_{8,-2,-1}(x)=x^{8}+4 x^{6}-8 x^{2}-2
$$

hence

$$
H_{4,-2,-1}(x)=x^{4}+4 x^{3}-8 x-2=\left(x^{2}+2 x\right)^{2}-4\left(x^{2}+2 x\right)-2
$$

so $H_{4,-2,-1}$ is decomposable.

Question. It is easy to see that $f_{9, B, a}$ is decomposable if and only if $B=2$ (provided $a \neq 0$ ). Does there exist decomposable $f_{n, B, a}$ for odd $n$, $a \neq 0$ and $B \neq 2$ ?

We say that polynomials $f, g$ are associated if there exist linear polynomials $L, M$ such that $g=L \circ f \circ M$. From (2.2) it follows that every Dickson 
polynomial $D_{n, a}$ with $a \neq 0$ is associated with some Dickson polynomial of the form $D_{n, 1}$.

Lemma 2 Assume that $f, g$ are associated polynomials. Then:

i) If $f, g$ are odd then

$$
g(x)=\alpha f(\gamma x)
$$

ii) If $f, g$ are even then

$$
g(x)=\alpha f(\gamma x)+\beta
$$

(for some constants $\alpha, \beta, \gamma$ ).

Proof. Let us prove i). By $g(x)=\alpha f(\gamma x+\delta)+\beta$ and the fact that $f, g$ are odd, we get the identity

$$
f(\gamma x-\delta)=f(\gamma x+\delta)+\frac{2 \beta}{\alpha} .
$$

If $\operatorname{deg} f=1$, then the result is trivially true. Thus we may assume that $f$ is not linear. Using the Taylor expansion around $\gamma x$ and nonlinearity of $f$ we get $\delta=0$, which infers $\beta=0$. One may prove ii) similarly.

Lemma 3 Assume that $f_{n, B, a}$ is associated with $D_{n, b}$ with $n \geq 5$. Then $a=b=0$ or $B=2$.

Proof. Using the results and notation from Lemma 2, we get:

$$
\begin{aligned}
\alpha \gamma^{n} & =1, \\
\alpha \gamma^{n-2}(B+n-2) b & =n a, \\
\alpha \gamma^{n-4} b^{2} \frac{(n+(B-2) 2)(n-3)}{2 !} & =\frac{n(n-3)}{2 !} a^{2} .
\end{aligned}
$$

We see that $a \neq 0$ if and only if $b \neq 0$. If $a \neq 0$ we get

$$
\gamma^{2}=\frac{(B+n-2) b}{n a} \quad \text { and } \quad \gamma^{4}=\frac{(n+(B-2) 2) b^{2}}{n a^{2}},
$$

hence $(B+n-2)^{2}=n(n+(B-2) 2)$, i.e. $B=2$.

We will need the information when our polynomials $f_{n}=f_{n, B, a}$ have only simple roots. Very precise information about the roots of polynomials 
$f_{n}$ may be obtained using known results on (quasi) orthogonal polynomials. Note that the polynomials $f_{n}$ satisfy a three term recurrence relation:

$$
x p_{n}(x)=\beta_{n} p_{n+1}(x)+\gamma_{n} p_{n}(x)+\delta_{n} p_{n-1}(x), \quad p_{0}(x)=1, p_{-1}(x)=0,
$$

with $\beta_{n}=1, \gamma_{n}=0, \delta_{1}=a B, \delta_{n}=a$ for $n \geq 2$. Namely, it is clear that for $n \geq 1$ we have $f_{n}=p_{n}$. By Favard's Theorem, the polynomials $f_{n}$ are quasi-definite orthogonal polynomials, and if $B>0, a>0$ they are positive definite (see $[4,21])$.

Theorem 4 Polynomials $f_{n, B, a}$ with $a, B \in \mathbb{R}$ and $a \neq 0$ have simple roots, except if $B=0$ and $n=2 k$ (then $x=0$ is a double root), or if $B=-1 / k$ and $n=2 k+1$ (then $x=0$ is a triple root).

Furthermore, let $\varepsilon=0$ if $n$ is even, while $\varepsilon=-1 / k$ if $n=2 k+1$. If $B \geq \varepsilon, a>0$, then all roots are real, if $B \geq \varepsilon, a<0$, then all roots are imaginary, if $B<\varepsilon, a>0$, then $n-2$ roots are real and two roots are imaginary conjugates, and if $B<\varepsilon, a<0$, then $n-2$ roots are imaginary and two roots are real.

Proof. According to Lemma 1 iii), it suffices to prove the statement for the case $a=1$.

If $B>0$, then $f_{n}$ is a positive definite orthogonal polynomial, and therefore all roots of $f_{n}$ are real and simple (see e.g. [21, Theorem 3.1.1]).

If $B<0$, then $f_{n}$ is a quasi-definite orthogonal polynomial. By a theorem of Gilbert [8] (see also [4, Theorem 2.4.6]), the roots of $f_{n}$ are all real or purely imaginary. Furthermore, the roots are simple, with the possible exception of a triple zero at $x=0$. However, the triple zero at $x=0$ is possible only when $n$ is odd, say $n=2 k+1$, and the coefficient with $x^{1}$, which is $(B k+1)(-a)^{k}$, is equal to zero, i.e. when $B=-1 / k$.

From a theorem of Veselić [22] (see also [4, Theorem 2.4.1]), it follows that $f_{n}$ has at least $n-2$ real roots. Since the coefficients with $x^{0}$ and $x^{1}$ are $B(-1)^{k}$ (for $n=2 k$ ) and $(B k+1)(-1)^{k}$ (for $n=2 k+1$ ), resp., we see that the product of all zeros of polynomials $f_{2 k}(\sqrt{x})$, resp. $f_{2 k+1}(\sqrt{x}) / \sqrt{x}$, is negative for $n$ even, while has the same sign as $B k+1$ for $n$ odd. Thus, if $n$ is even, or $n$ is odd and $B<-1 / k$, then at least one of these zeros is negative, which implies that the two remaining roots of $f_{n}$ are imaginary conjugates.

The case $B=0$ follows from $f_{n, 0, a}=x f_{n-1,1, a}$ and the results already proved for $B=1$. 
Lemma 4 Let $C$ be an algebraic curve of genus $\mathfrak{g}$, defined over the field of complex numbers with affine equation

$$
y^{m}=f(x)
$$

where $f$ is a polynomial of degree $n$ and with simple roots. Then

$$
2 \mathfrak{g}-2=m n-m-n-\operatorname{gcd}(m, n) .
$$

Proof. See, for example, [16, Proposition VI.3.1].

In the following theorem we illustrate an application of previous results on Diophantine equations of form $f(x)=g(y)$.

We continue to use notation $B=\frac{\mu}{d}$, introduced in Section 2. Similarly, for a rational number $C$, we set $C:=\frac{\nu}{D}$, where $\nu, D$ are co-prime integers and $D>0$, and if $C=0$ then we set $\nu=0$ and $D=1$.

TheOrem 5 Let us put $f=f_{n, B, a}$ and $g=f_{m, C, b}$. Assume that $\operatorname{gcd}(d, n)=$ $\operatorname{gcd}(\mu-2 d, n)=\operatorname{gcd}(D, m)=\operatorname{gcd}(\nu-2 D, m)=1$ and that $m, n$ are odd and different from 1.

Then the equation $f(x)=g(y)$ has only finitely many rational solutions with a bounded denominator, except if $f=g$ or $a=b=0$.

Proof. In this settings $f, g$ are indecomposable, by Theorem 3, and so, by Theorem 1, if the equation $f(x)=g(y)$ has infinitely many rational solutions with a bounded denominator, then $f$ and $g$ are associated with the polynomials from a standard pair, or $f$ and $g$ are associated to each other. By Lemma 2 and Lemma 3, this is impossible provided $m, n \geq 5$ and $m \neq n$, and $a \neq 0$ and $b \neq 0$.

However, if $m=n$, then we have $g(x)=f(\gamma x+\delta)$. By Lemma 2, it follows that $\delta=0$ and $\gamma=1$, and hence $f=g$.

Furthermore, if $b=0$, then for $a \neq 0$ we have $g(x)=x^{m}$, and so, by Lemma 4 , the genus of $f(x)=y^{m}$ is greater than 1 (note that, by Theorem 4 , $f$ is without multiple roots). Hence the equation has finitely many rational solutions.

Assume now that $m=3$ and $n \geq 5$, and that the equation $f(x)=g(y)$ has infinitely many rational solutions with a bounded denominator. We have to look at standard pairs of the first kind, hence $g$ should be associated with $x^{3}$. By Lemma 2, we see that $g(x)=\alpha(\gamma x)^{3}=x^{3}$, and by Theorem 4 and Lemma 4 the equation $y^{3}=f(x)$ has genus greater than 1 , and therefore it has only finitely many rational solutions. 
The remaining case is $m=n=3$. We look at the equation

$$
y^{3}-b(C+1) y=x^{3}-a(B+1) x
$$

such that $f \neq g$, i.e. $b(C+1) \neq a(B+1)$. It is easily seen that no standard pair is possible in this situation, except the trivial pair $(x, p(x))$ with $p$ linear. Hence, we must have $g(x)=f(\gamma x+\delta)$ (provided the equation has infinitely many rational solutions), and by Lemma 2 we obtain $\delta=0$ and $\gamma=1$, a contradiction.

REMARK 2 One may prove a similar result if $n$ is odd and $m$ even, as well as if $m, n$ are both even. The equation has finitely many rational solutions with a bounded denominator, except in few exceptional (trivial) cases.

In particular, for $B=C=1$ we obtain the result, already proved in [7] and [5], that the equation $G_{n}(x)=G_{m}(y)$, where $G_{n}:=f_{n-1,1, a}(a \neq 0)$ are generalized Fibonacci polynomials, has only finitely many integer solutions for $m, n \geq 4, m \neq n$.

A similar result can be proved for $B=C=3$. Let $V_{n}:=f_{n, 3, a}(a \neq 0)$. According to Remark $1, V_{n}$ is indecomposable for $n$ odd and has the unique decomposition $V_{n}(x)=H_{n / 2}\left(x^{2}\right)$ (up to equivalence) for $n$ even. As in [5, Theorem 5.1 and Corollary 5.1], it follows that the equation $V_{n}(x)=$ $V_{m}(y)$ has only finitely many integer solutions for $m, n \geq 3, m \neq n$, unless $n=2 m$ and $H_{n}(x)=V_{n}(\gamma x+\delta)$. But the last possibility can be excluded by considering the coefficients of $x^{n}, x^{n-1}, x^{n-2}$ and $x^{n-3}$ in the equality $H_{n}(x)=V_{n}(\gamma x+\delta)($ as in $[5$, Theorem 4.1]).

Acknowledgement: The authors would like to thank the referee for several very useful suggestions.

\section{References}

[1] Yu. Bilu, B. Brindza, P. Kirschenhofer, Á. Pintér and R. F. Tichy, Diophantine equations and Bernoulli polynomials, (with an appendix by A. Schinzel), Compositio Math. 131 (2002), 173-188.

[2] Yu. Bilu, Th. Stoll and R. F. Tichy, Octahedrons with equally many lattice points, Period. Math. Hungar. 40 (2000), 229-238.

[3] Yu. Bilu and R. F. Tichy, The Diophantine equation $f(x)=g(y)$, Acta Arith. 95 (2000), 261-288. 
[4] M. V. DeFazio, On the Zeros of Some Quasi-definite Orthogonal Polynomials, PhD Dissertation, York University, Toronto, 2001.

[5] A. Dujella and I. Gusić, Indecomposability of polynomials and related Diophantine equations, Q. J. Math. 57 (2006), 193-201.

[6] A. Dujella, I. Gusić and R. F. Tichy, On the indecomposability of polynomials, Österreich. Akad. Wiss. Math.-Natur. Kl. Sitzungsber. II 214 (2005), 81-88.

[7] A. Dujella and R. F. Tichy, Diophantine equations for second order recursive sequences of polynomials, Quart. J. Math. Oxford Ser. (2) 52 (2001), 161-169.

[8] G. S. Gilbert, Zeros of symmetric, quasi-definite, orthogonal polynomials, J. Math. Anal. App. 157 (1991), 346-350.

[9] R. L. Graham, D. E. Knuth and O. Patashnik, Concrete Mathematics, Addison-Wesley, Reading, 1994.

[10] P. Kirschenhofer and O. Pfeiffer, Diophantine equations between polynomials obeying second order recurrences, Period. Math. Hungar. 47 (2003), 119-134.

[11] M. Kulkarni and B. Sury, A class of Diophantine equations involving Bernoulli polynomials, Indag. Math. 16 (2005), 51-65.

[12] M. Kulkarni and B. Sury, Diophantine equations with Bernoulli polynomials, Acta Arith. 116 (2005), 25-34.

[13] C. Rakaczki, On the diophantine equation $S_{m}(x)=g(y)$, Publ. Math. Debrecen 65 (2004), 439-460.

[14] A. Schinzel, Selected Topics on Polynomials, University of Michigan Press, 1982.

[15] A. Schinzel, Polynomials with Special Regard to Reducibility, Cambridge University Press, 2000.

[16] H. Stichtenoth, Algebraic Function Fields and Codes, Springer-Verlag, Berlin, 1993.

[17] Th. Stoll, Finiteness Results for Diophantine Equations Involving Polynomial Families, PhD Dissertation, Technische Universität Graz, 2003.

[18] Th. Stoll and R. F. Tichy, Diophantine equations for classical continuous orthogonal polynomials, Indag. Math. 14 (2003), 263-274.

[19] Th. Stoll and R. F. Tichy, Diophantine equations involving general Meixner and Krawtchouk polynomials, Quaest. Math. 27 (2004), 1-11.

[20] Th. Stoll and R. F. Tichy, Diophantine equations for Morgan-Voyce and other modified orthogonal polynomials, preprint. 
[21] G. Szegö, Orthogonal Polynomials, American Mathematical Society, Providence, 2003.

[22] K. Veselić, On real eigenvalues of real tridiagonal matrices, Lin. Alg. Appl. 27 (1979), 167-171.

Andrej Dujella

Department of Mathematics

University of Zagreb

Bijenička cesta 30

10000 Zagreb, Croatia

E-mail address: duje@math.hr

Ivica Gusić

Faculty of Chemical Engineering and Technology

University of Zagreb

Marulićev trg 19

10000 Zagreb, Croatia

E-mail address: igusic@fkit.hr 